\title{
An Adverse Case of Spinal Tumor Embolization with Gelfoam in a Patient Whose Vertebral Artery Has Shared Origin with Ipsilateral Costocervical Trunk
}

\author{
Han Jun Kim, MD (D; Jung Hoon Han, MD (D; Chi Kyung Kim, MD, PhD (D; Kyungmi Oh, MD, PhD (D) \\ Department of Neurology, Korea University Guro Hospital, Korea University College of Medicine, Seoul, Korea
}

Tumor embolization is performed before surgical excision. The Gelfoam temporarily occludes the vessels supplying the tumor by facilitating thrombus formation. We report an adverse case of Gelfoam embolization in a patient with a certain vascular anatomy. A 75-year-old man previously diagnosed with lung cancer in 2015 was admitted to Korea University Guro Hospital. He had bilateral arm paresthesia and lower extremity weakness that had progressed for 2 weeks. Cervical spine magnetic resonance imaging revealed a metastatic pathologic fracture of the C6 vertebral body and subsequent cord compression. A C6 corpectomy was scheduled, and preoperative spinal tumor embolization was planned. Angiography revealed that the left deep cervical artery (DCA) and the ipsilateral vertebral artery shared origin. Two Nester coils were positioned at the right distal DCA, one at the left DCA, and two at the left proximal DCA. Gelfoam was infused in each location. However, the patient's mental status worsened after the left DCA embolization. A diffusion-weighted image showed diffuse cytotoxic edema in the posterior circulation without significant lesions on magnetic resonance angiography. In Gelfoam embolization, special attention is required with neurological monitoring when maneuvering DCA if it has a nearby entrance with a vertebral artery.

\section{J Neurosonol Neuroimag 2021;13(2):96-79}

Key Words: Gelatin sponge, absorbable; Spinal neoplasms; Embolization, therapeutic
Received: November 10, 2021 Revised: December 6, 2021 Accepted: December 8, 2021

Correspondence:

Kyungmi Oh, MD, PhD

Department of Neurology, Korea University Guro Hospital, Korea University College of Medicine, 148 Gurodong-ro, Guro-gu, Seoul 08308, Korea

Tel: +82-2-2626-1250

Fax: +82-2-2626-2249

E-mail: okyungmi@korea.ac.kr
Tumor embolization is occasionally performed before surgical excision. It is a minimally invasive vascular procedure wherein the feeding vessels of tumors are occluded using interventional techniques. With this preoperative process, reduced intraoperative blood loss and improved visibility of tumor lesions can be anticipated. ${ }^{1}$ The Gelfoam is a biologic substance derived from purified skin gelatin, ${ }^{2}$ which can result in temporary occlusion after intravascular injection by facilitating thrombus formation. ${ }^{3}$ Among various adverse events of Gelfoam embolization, embolic events to untargeted vessels, which cause unexpected organ damage, is a major consideration when performing the procedure. ${ }^{4}$ We report an unpredicted adverse case of Gelfoam embolization to a spinal tumor with a particu- lar vascular anatomy.

\section{CASE}

A 75-year-old male patient was admitted to the Department of Orthopedic Surgery in Korea University Guro Hospital in June 2021. The patient complained of bilateral arm paresthesia and lower extremity weakness, which progressed within 2 weeks and led him to become bedridden. He was previously diagnosed with non-small cell lung carcinoma in 2015 at the Department of Pulmonology in Korea University Guro Hospital and was undergoing chemotherapy. The latest chemotherapy was performed with docetaxel about 1 
month before the current admission without recognizable neurological symptoms. Upon admission, he had stable vital signs and routine laboratory tests, including inflammatory markers and coagulation tests, were within the normal range. A new pathologic fracture at the 6th cervical vertebral (C6) body along with central cord compression was found on whole spine magnetic resonance imaging compared to the one performed approximately seven months earlier (Fig. 1A, B). Emergent C6 vertebral corpectomy with fixation was scheduled, and preoperative spinal tumor embolization was planned to reduce blood loss intraoperatively. While undergoing embolization, $100 \mu \mathrm{g}$ of fentanyl was slowly infused from the beginning for pain management, but the patient repeatedly complained of back pain. On the control angiogram, radiocontrast staining of the $\mathrm{C} 6$
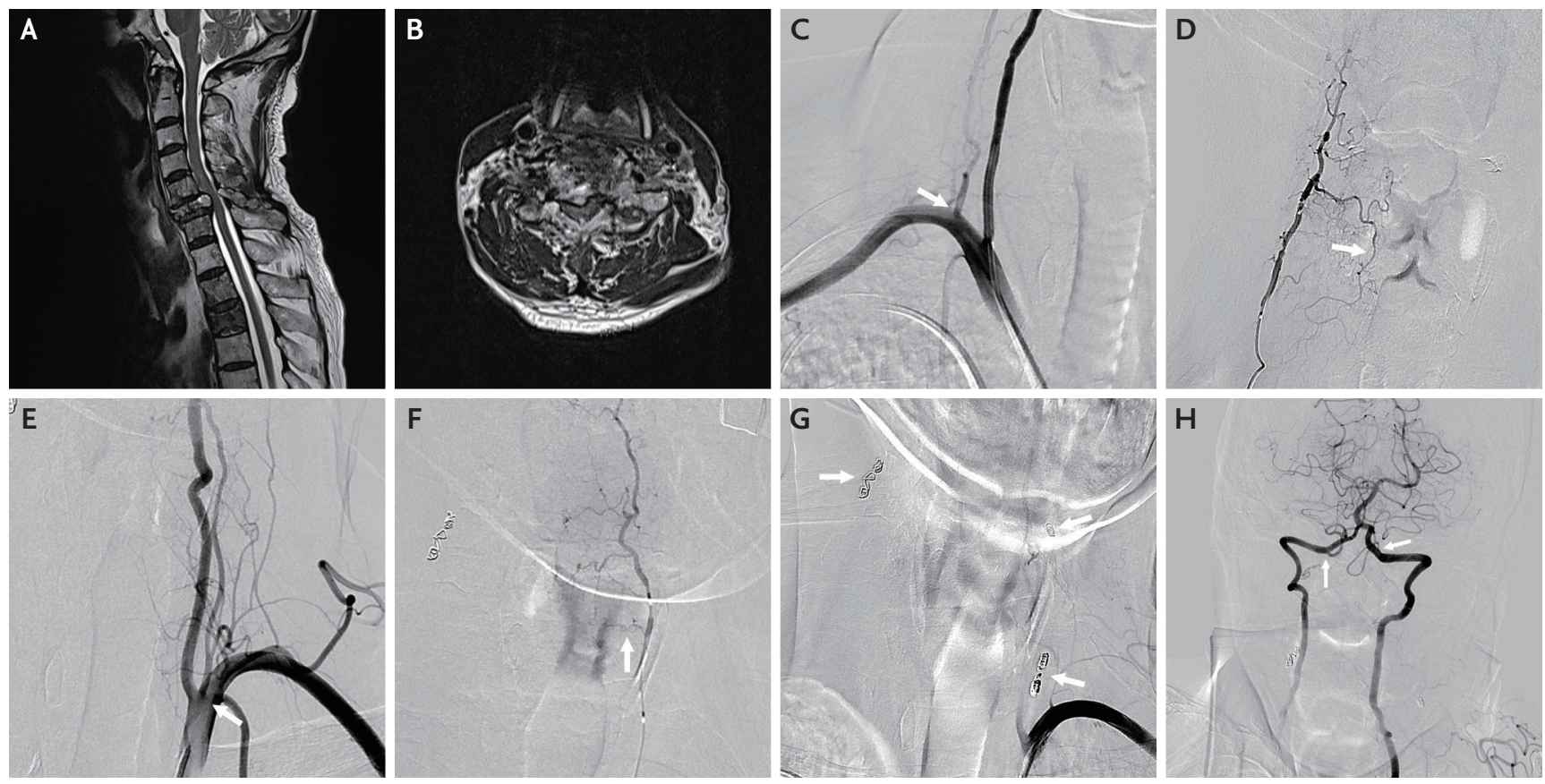

FIG. 1. (A) Metastatic tumor involving $\mathrm{C}_{5}-7$ vertebral body and pathologic fracture in C6 vertebral body. (B) Consequent central cord compression in C6 level. (C) Right vertebral artery angiogram showing some distance between the origins of right vertebral artery (VA) and ipsilateral costocervical trunk (arrow). (D) Right DCA angiogram identifying feeding branches (arrow) to the metastatic spinal tumor with contrast staining. (E) Left subclavian angiogram demonstrating left DCA having the common origin with left VA (arrow). (F) Left DCA angiogram showing feeding branches (arrow) to the tumor with contrast staining. (G) Location of Nester coils at cerebral angiography. Two at right distal DCA, one at left distal DCA, and two at left proximal DCA (arrows). (H) Final angiography from left VA including retrograde flow to the right distal VA. Both posterior inferior cerebellar arteries originated from their ipsilateral distal vertebral arteries (arrows). There was no abnormality indicating stenosis, occlusion, or dissection.
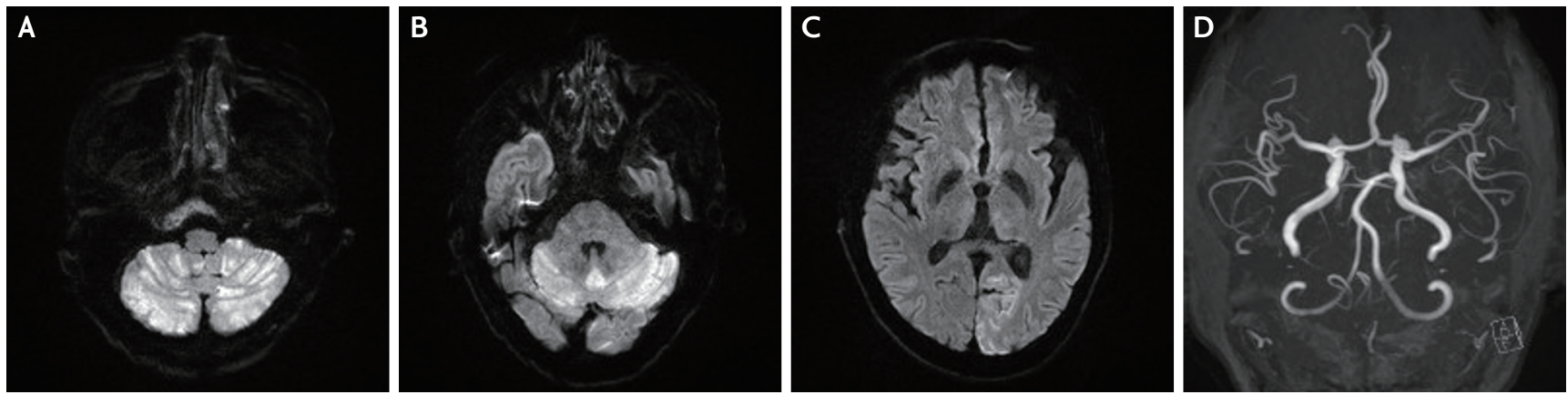

FIG. 2. (A-C) Diffusion-weighted image showing multifocal, diffuse cytotoxic edema at posterior circulation. (D) Non-enhance intracranial magnetic resonance angiography without significant pathological findings. 
vertebral tumor was found in the bilateral deep cervical artery (DCA) (Fig. 1D-F). There were no significant feeding arteries and pathologic findings encountered from either side of the vertebral arteries (VA). Angiography from the right VA revealed some distance between the origins of the VA and the ipsilateral costocervical trunk (Fig. 1C). However, on angiography of the left subclavian artery, there was an uncommon sharing of origins between the left VA and ipsilateral costocervical trunk (Fig. 1E). Two Nester coils (Cook, Bloomington, IN, USA) were positioned at the right distal DCA, one at the left distal DCA, and the last two at the left proximal DCA (Fig. 1G). Gelfoam (NEXTBIOMEDICAL, Incheon, Korea) sponge particle caliber of $350-500 \mu \mathrm{m}$ was infused at each location. After each DCA embolization, we performed angiography at the orifice of the ipsilateral VA and found no abnormalities in either case (Fig. $1 \mathrm{H}$ ). During embolization of the right DCA, the patient's alertness was maintained with frequent pain complaints. However, after finishing the left DCA embolization, the alertness abruptly deteriorated. Two neurologists (J.H., H.J.) performed a thorough neurological examination, and a down-beating nystagmus with a large amplitude was found. Under the suspicion of cerebellar and adjacent lesions, diffusion-weighted imaging (DWI) was taken immediately, showing widespread cytotoxic edema only in the posterior circulation without any significant steno-occlusive lesion on concurrent intracranial magnetic resonance (MR) angiography (Fig. 2).

\section{DISCUSSION}

The extracranial variation in VA is very rare. ${ }^{5}$ Particularly, only about $5 \%$ in left VA has been reported. ${ }^{6}$ In most cases, it remains asymptomatic, whereas complications could occur during the interventional process.? It is reported that the diameter of the VA and basilar artery (BA) reach 2.9-3.4 and 2.7-4.3 $\mathrm{mm}$, respectively. ${ }^{8} \mathrm{In}$ contrast, VA and BA perforators have a relatively narrow diameter of $210-940 \mu \mathrm{m} .{ }^{9}$ Therapeutic embolization is used to occlude feeding vessels of tumors or control bleeding in trauma patients and aims to provide a structure in which thrombus can occur, resulting in a permanent or temporary occlusion of the target ves- sels. ${ }^{6}$ Gelfoam is the most commonly used temporary embolic agent, and the duration of occlusion takes days to weeks. ${ }^{6,10}$ It has the advantage that it can reach distal vessels for occlusion owing to its small particle size and can be used for strengthening of formerly located microcoils. ${ }^{6}$ Despite these benefits, Gelfoam can inadvertently reflux into off-target vessels and cause unwanted occlusion. ${ }^{10}$

In this case, sudden neurological deterioration occurred after embolization of the bilateral DCA. In terms of the embolization process, only the distal part of the vessel was embolized in the right DCA. However, on the left side, coil and Gelfoam embolization were performed down to the proximal part of the left DCA, which is close to the origin of the left VA (Fig. $1 \mathrm{G}$ ). Considering that the patient's neurological deterioration occurred immediately after finishing left DCA embolization, reflux of the Gelfoam material was suspected.

On DWI, multifocal lesions were identified in a wide area of the bilateral posterior circulation territory (Fig. 2A-C). It is not clear whether the Gelfoam reflux from a single VA indeed caused such diffuse, bilateral lesions without leaving any pathologic findings on angiography. When referring to the final angiogram from the left VA (Fig. $2 \mathrm{H}$ ), there is a possibility that the reflux ran to the contralateral distal VA and both posterior inferior cerebellar arteries. In addition, the small diameter of Gelfoam particles could enable them to disperse into the distal tiny branches or perforating arteries, which are not identifiable even in digital subtraction angiography (DSA). This might further explain why the pattern of infarctions was multifocal rather than territorial and included focal lesions from small vessel territories such as the brainstem and thalamus (Fig. 2). Despite these assumptions, given the clinical situations of advanced cancer and incomplete evaluation of other embolization sources, possibilities such as cancer-related stroke cannot be excluded.

We report a case of unpredicted complications of Gelfoam embolization in a patient with a common origin of the left VA and costocervical trunk. Given that detection of Gelfoam particles may not be possible even with DSA, concurrent neurological monitoring should be performed along with interventional caution to minimize and ultimately prevent complications from the procedure. 


\section{Ethics Statement}

The study's protocol was reviewed and approved by the Institutional Review Board (IRB) of Korea University Medical Center, Guro Hospital (IRB No. 2011GRo218). Informed consent was waived.

\section{Availability of Data and Material}

None.

\section{Acknowledgments}

This research was supported by R\&D support grant (K2108031) of Korea University Guro Hospital, Republic of Korea.

\section{Sources of Funding}

None.

\section{Conflicts of Interest}

No potential conflicts of interest relevant to this article was reported.

\section{REFERENCES}

1. Nair S, Gobin YP, Leng LZ, Marcus JD, Bilsky M, Laufer I, et al. Preoperative embolization of hypervascular thoracic, lumbar, and sacral spinal column tumors: technique and outcomes from a single center. Interv Neuroradiol. 2013;19:377-385.

2. Abada HT, Golzarian J. Gelatine sponge particles: handling characteristics for endovascular use. Tech Vasc Interv Radiol. 2007;10:257-260.

3. Pereira BM, Bortoto JB, Fraga GP. Topical hemostatic agents in surgery: review and prospects. Rev Col Bras Cir. 2018;45:e1900.

4. Medsinge A, Zajko A, Orons P, Amesur N, Santos E. A casebased approach to common embolization agents used in vascular interventional radiology. AJR Am J Roentgenol. 2014;203:699-708.

5. Lopera JE. Embolization in trauma: principles and techniques. Semin Intervent Radiol. 2010;27:14-28.

6. Haughton VM. The normal and anomalous aortic arch and brachiocepalic arteries. Angiography.1974:1149-1150.

7. Tokuyama K, Kiyosue H, Baba H, Asayama Y. Anomalous origin of the right vertebral artery. Appl Sci. 2021;11:8171.

8. Mehinovic A, Isakovic E, Delic J. Variations in diameters of vertebro-basilar tree in patients with or with no aneurysm. Med Arch. 2014;68:27-29.

9. Chen L, Chen E, Chotai S, Tian X. An endovascular approach to ruptured aneurysms of the circumferential branch of the basilar artery. J Clin Neurosci. 2012;19:527-531.

10. Levin DC, Beckmann CF, Hillman B. Experimental determination of flow patterns of gelfoam emboli: safety implications. AJR Am J Roentgenol. 1980;134:525-528. 